\title{
Modeling of a Gyro-stabilized Helicopter Camera System Using Artificial Neural Networks
}

\author{
Nicholas Layshot, Xiao-Hua Yu
}

\begin{abstract}
On-board gimbal systems for camera stabilization in helicopters are typically based on linear models. Such models, however, are inaccurate due to system nonlinearities and complexities. As an alternative approach, artificial neural networks can provide a more accurate model of the gimbal system based on their non-linear mapping and generalization capabilities.

This paper investigates the applications of artificial neural networks to model the inertial characteristics (on the azimuth axis) of the inner gimbal in a gyro-stabilized multi-gimbal system. The neural network is trained with time-domain data obtained from gyro rate sensors of an actual camera system. The network performance is evaluated and compared with measurement data and a traditional model. Computer simulation results show the neural network model fits well with the measurement data and significantly outperforms the traditional model.
\end{abstract}

\section{INTRODUCTION}

$\mathrm{O}$ N-BOARD camera systems have been widely used in many applications such as fire detection, law enforcement, television news programs, as well as military applications. The camera is often mounted in a roll tube that is contained within a two-axis "inner" gimbal which is often mounted inside another two-axis "outer" gimbal.

The outer two-axis gimbal allows the camera to be panned 360 degrees in azimuth and over 180 degrees in elevation. The inner two-axis gimbal has a limited range of movement (a few degrees of travel), but has a much larger bandwidth than the outer gimbal. This higher bandwidth gives the inner gimbal the ability to counteract the smaller amplitude, higher frequency vibrations of the mounting surface (such as a helicopter). A typical two-axis double gimbal system is shown in Fig. 1.

The system of interest in this paper is the inner gimbal azimuth axis system. The transfer function of such system is typically derived from either FEA (finite element analysis) or modal analysis [1]. In fact, the model of a gyro-stabilized gimbal system is very difficult to obtain due to its complicated non-symmetrical mechanical design, nonlinear structural dynamics, and the many torque disturbances involved in the system. Therefore, many nonlinear effects are usually not considered in a typical multi-gimbal system model, including the products-of-inertia, kinematic coupling, as well as gyroscopic effects [2]. In addition, though gimbals are often supported by a firm structure, they are usually exposed to or operated in a mobile and dynamic environment. For example, in a flying aircraft, wind can induce high frequency vibrations into the gimbal system. In a marine environment, water vapor and moisture leakage are additional potential sources of nonlinear frictions. In a ground vehicle, the random shock and resonance can be very severe. To better control the performance of a gimbal system under various environments, an accurate and adaptive model must be developed.

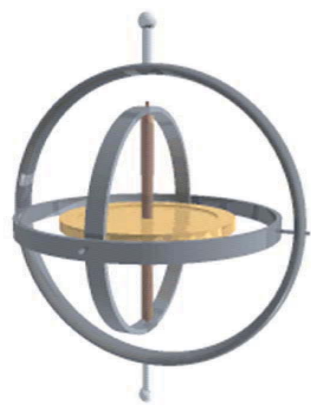

Fig. 1. The two-axis multi-gimbal system

In this research, artificial neural network is employed to model the inner gimbal azimuth axis system. The torque disturbances and nonlinearities considered in this paper include the bearing friction Coulomb model effects, kinematic coupling effects, and cable nonlinearities. The neural network is trained with time-domain data obtained from gyro rate sensors of an actual camera system. The network performance is evaluated and compared with measurement data and a traditional linear model. With the nonlinear mapping ability and adaptive learning ability, the neural network model fits well with the measurement data and significantly outperforms the traditional model.

\section{THE InNER Gimbal AZIMUCH AXIS SYSTEM}

The overall system block diagram of the inner gimbal azimuth axis system studied in this research is shown in Fig. 2. The input is the voltage (in volts) to the motor/amplifier that controls the inner azimuth gimbal. This input drives a "torquer" motor which consists of a coil (attached to the outer gimbal) and a magnet (attached to the inner gimbal). The output is the relative azimuth inertial rate (radians/second) between the inner and the outer gimbal that 
can be measured from a gyro rate sensor located on the payload (e.g., a camera).

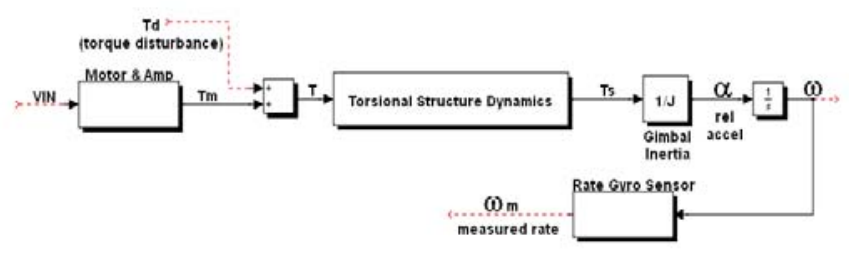

Fig. 2. The inner gimbal azimuth axis system

The torque disturbance $T_{d}$ for an inner gimbal may be caused by many factors such as the sliding or rubbing frictions from the seals and bearings, the payload unbalance, the gyroscopic torques from gyro rate sensors, on-board shaking forces, actuator and gear reactions, cable torques, spring torques, structural bending, actuator cogging, and ripple torques, etc. [1]. Due to the nonlinear and random nature of the above issues, torque disturbance is very difficult to model.

The Dahl model is one of the most commonly used dynamic friction model for ball bearings. As shown in Fig. 2 , the relationship between the torque on the bearing (y-axis) and the bearing angle (x-axis) is highly nonlinear with hysteresis:

$$
\begin{aligned}
& \frac{d T_{B}}{d \theta}=\gamma\left[T_{s}-T_{B} \cdot \operatorname{sgn}(\dot{\theta})\right]^{\beta} \\
& \gamma=\frac{s}{T_{s}-\left|T_{p}\right|}
\end{aligned}
$$

where $T_{B}$ is the torque on the bearing, $\theta$ is the bearing angle, $s=$ slope at reversal (reversal of torque $T_{B}$ direction), $T_{s}$ is the maximum Dahl friction torque, $T_{p}$ is the value of torque at turnaround or reversal, and $\beta$ (values range $\geq 0$ ) is the parameter that determines the shape of the stress-strain curve (i.e., torque vs. angle curve) [3].

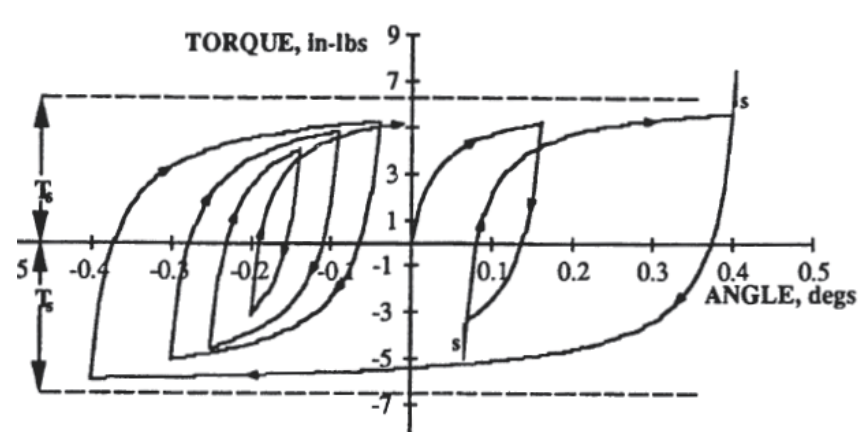

Fig. 2. Dahl Friction Model Example (torque vs. angle) ([1])

\section{THE NEURAL NETWORK MODEL}

In this section, a multi-layer feedforward artificial neural network (ANN) for modeling the inner gimbal azimuth axis system is discussed. The ANN model (shown in Fig. 3) has an input layer, an output layer, and one hidden layer. The inputs of neural network include the current and delayed inputs, as well as the delayed outputs of the inner gimbal azimuth axis system. That is, the neural network model is a multi-input, single-output system. The activation function for each hidden neuron is chosen as the hyperbolic tangent function:

$$
f(x)=\frac{1-e^{-x}}{1+e^{-x}}
$$

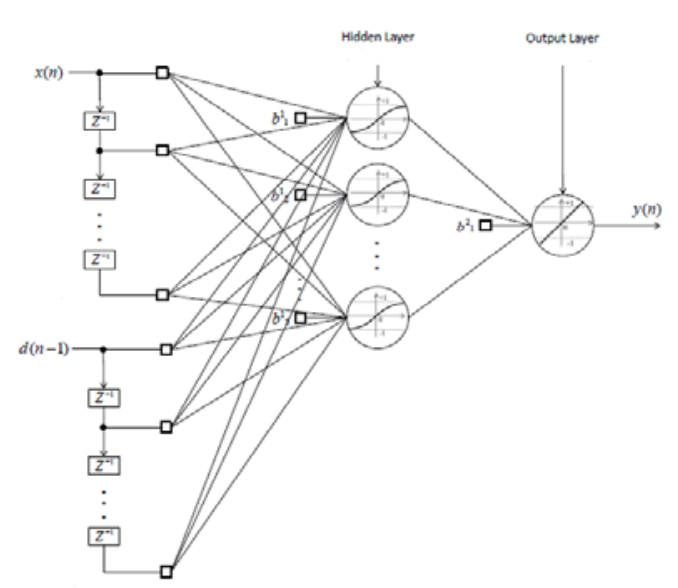

Fig. 3. The neural network model

The weights (including biases) of the hidden layer are initialized using the Nguyen-Widrow method [4]. The principle of this method is to approximate a nonlinear function as a union of piece-wise linear segments; and each of these segments is attributed by a neuron in the first layer of the network. It has been shown that the Nguyen-Widrow method can significantly reduce neural network training time on a large number of different problems [4]. The weights and bias of the output layer are initialized at random. All the weights are updated using the Levenberg-Marquardt algorithm to minimize the following objective function:

$$
J=\frac{1}{2 N} \sum_{i=1}^{N}[e(i)]^{2}=\frac{1}{2 N} \sum_{i=1}^{N}[d(i)-y(i)]^{2}
$$

Batch learning approach employed in this study, where $d$ is the desired output and $y$ is the output of neural network; $e$ is the output error; $i$ is the index of training pair; and $N$ is the batch size.

$$
\begin{aligned}
& W(k+1)=W(k)+\Delta W \\
& \Delta W=\left(J_{a}{ }^{T} J_{a}+\mu I\right)^{-1} J_{a}{ }^{T} e
\end{aligned}
$$

where $J_{a}$ is the first order derivative of the error function with respect to the neural network weight (also called the Jacobian matrix) $\frac{\partial e}{\partial W} ; \mu$ is a learning parameter, and $k$ is the index of iterations.

\section{Simulation Results}

In this section, the performance of the neural network 
model is evaluated and compared with the true measurement data and a traditional model. The training data and testing data are obtained from the time history measurement of a camera system developed by Axsys Technologies (a division of General Dynamics Advanced Information Systems), with the sampling frequency of $2500 \mathrm{~Hz}$.

First, the system response to a random input signal is recorded and then used to train the neural network model (20,000 training pairs). Then the trained neural network model is tested on various input signals and compared with the true system output data obtained from measurement. Fig. 4 shows the neural network output and the system output to a low frequency $(5 \mathrm{~Hz})$ signal, where the solid line represent the neural network output and the dashed line is for the true system response.
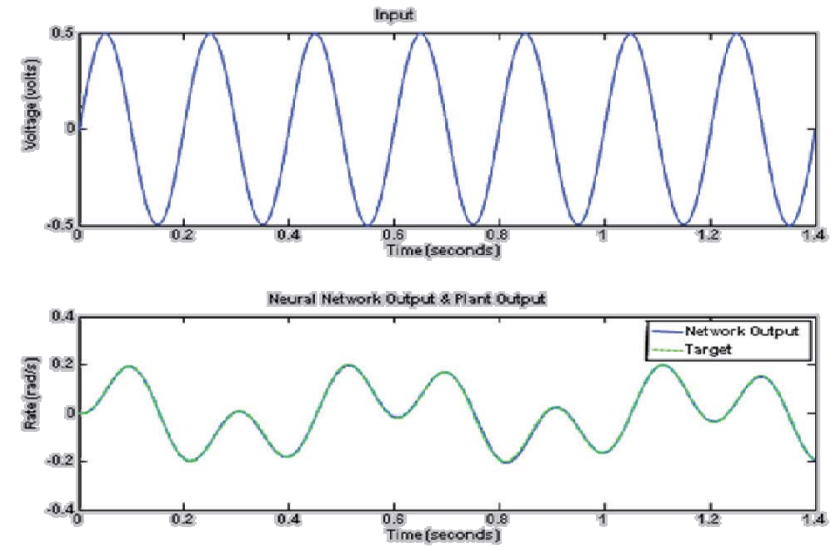

Fig. 4. The neural network output and system response to a $5 \mathrm{~Hz}$ sinusoidal signal

Fig. 5 illustrates the neural network output and the system output to a "multi-frequency" sinusoidal signal $(A=0.5)$ :

$$
\begin{aligned}
x(t)=A[\sin (10 \pi t)+\sin (30 \pi t)+\sin (40 \pi t) \\
+\sin (60 \pi t)+\sin (80 \pi t)+\sin (100 \pi t)]
\end{aligned}
$$

Finally, the neural network performance is tested using a "chirp" signal which has widely been used for system identification:

$$
x(t)=A \cos \left[\left(\omega_{1}+\frac{\left(\omega_{2}-\omega_{1}\right) t}{2 M}\right) t\right]
$$

where $\omega_{1}=0 \mathrm{~Hz}$ (DC), $\omega_{2}=1250 \mathrm{~Hz}$ (the highest frequency signal that satisfies the Nyquist sampling theorem for the sampling rate of $2500 \mathrm{~Hz}$ ), $0 \leq t \leq M$ (where $\mathrm{M}$ is the time period). The result is shown in Fig. 6; the zoomed in version and the input signal is shown in Fig. 7.
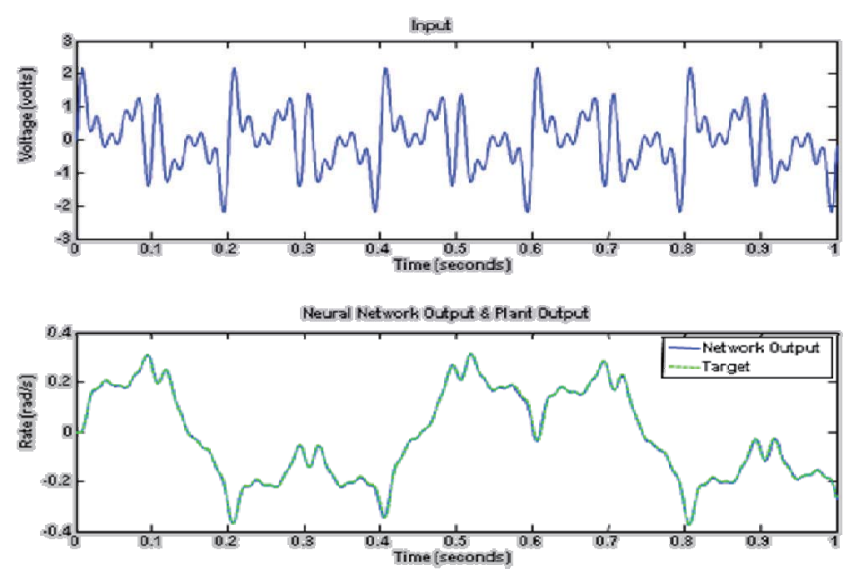

Fig. 5. The neural network output and system response to a "multi-frequency" sinusoidal signal

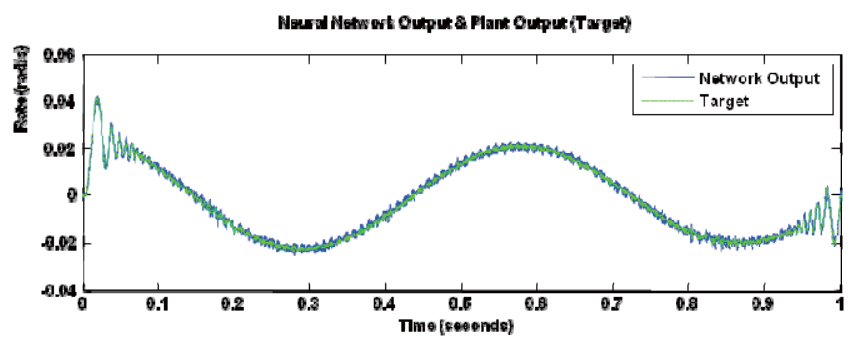

Fig. 6. The neural network output and system response to a "chirp" signal
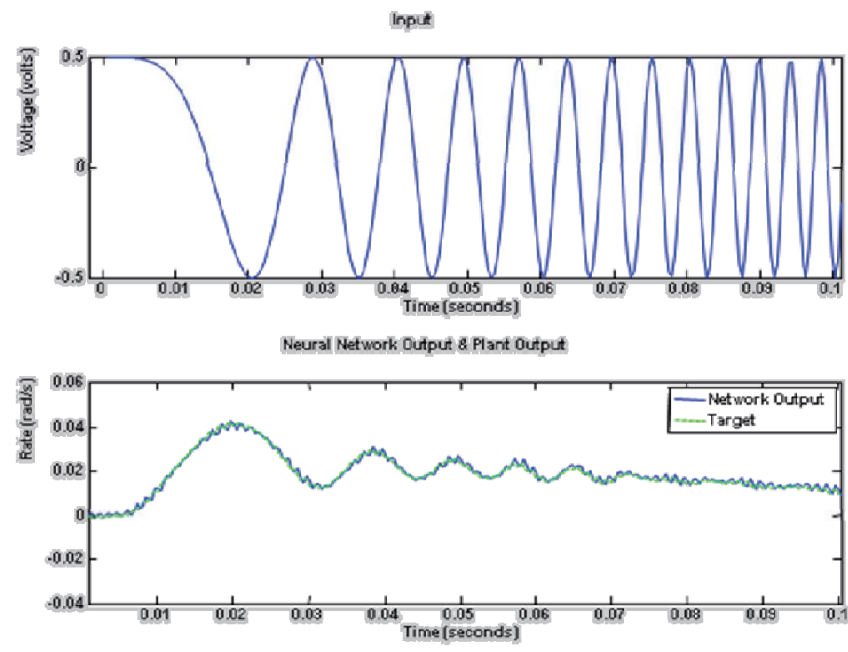

Fig. 7. The neural network output and system response to a "chirp" signal (zoomed in)

For a complete study, a conventional model for inner gimbal azimuth axis system is considered and its performance is investigated. Assume that the torsional structure is rigid; and the torque disturbance contains the 
spring torque and a dampening torque:

$$
T_{d}=-K_{s} \Delta \theta-c \dot{\theta}
$$

where $K_{s}$ is the spring constant; $\Delta \theta$ is the relative angular displacement between the inner and outer azimuth gimbal; $c$ is the system damping; and $\dot{\theta}$ is angular velocity. The Matlab Simulink model is shown in Fig. 8.

The motor/amplifier block shown in Fig. 2 consists of a current amplifier which drives a "torqer" motor consisting of a coil and magnet. The coil is attached to the outer gimbal while the magnet is attached to the inner gimbal. Fig. 9 shows the model of the motor/amplifier block.

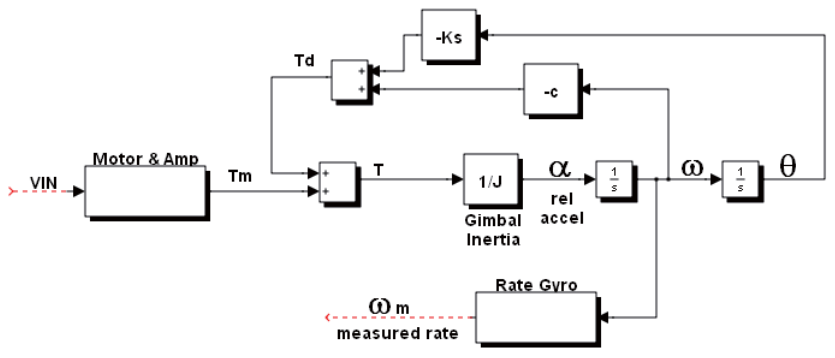

Fig. 8. Gimbal (rigid structure w/ spring \& dampening torque disturbances)

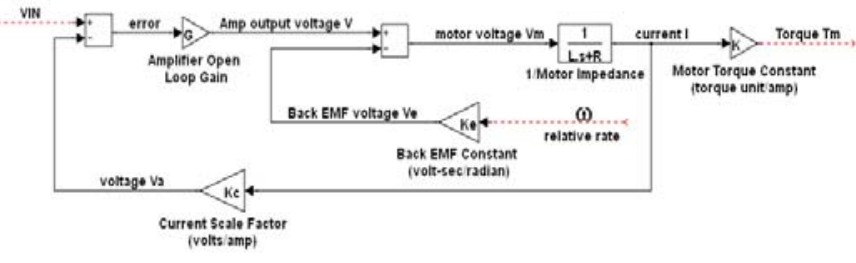

Fig. 9. Amplifier/motor model

The gyro rate sensor is illustrated in Fig. 10. The corner frequency of the gyro $\omega_{n} \approx 628(\mathrm{rad} / \mathrm{s})$ and the quality factor $\mathrm{Q} \approx 10$.

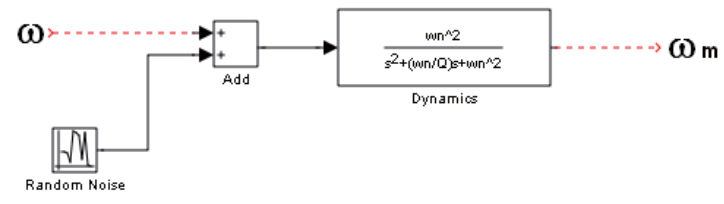

Fig. 10. Gyro rate sensor

The simulation results of the performance of the conventional model is illustrated in Fig. 11, 12, and 13.

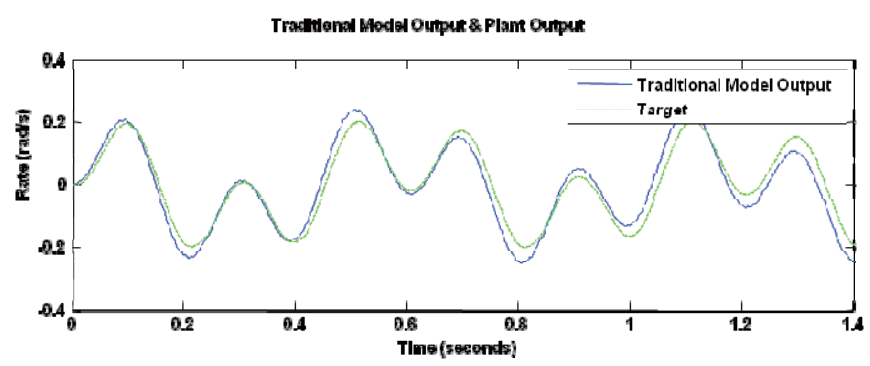

Fig. 11. The conventional model output and system response to a $5 \mathrm{~Hz}$ sinusoidal signal

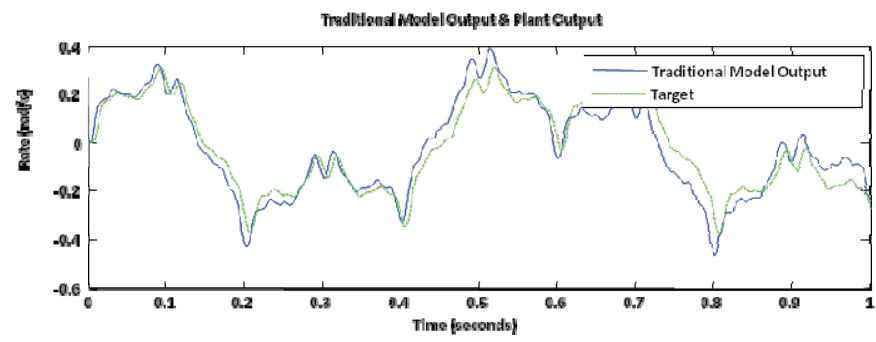

Fig. 12. The conventional model output and system response to a "multi-frequency" sinusoidal signal

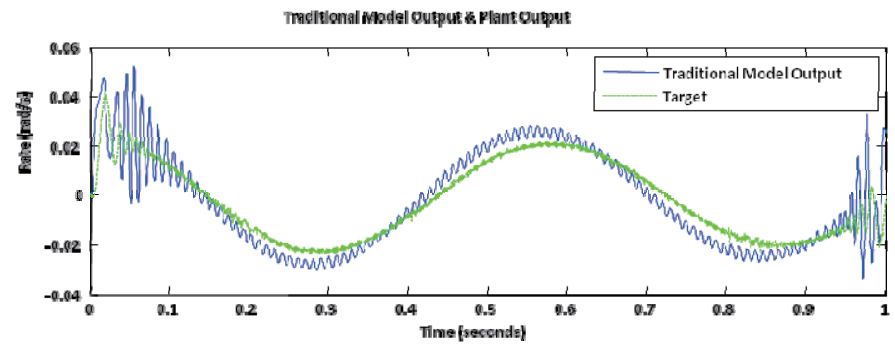

Fig. 13. The conventional model output and system response to a "chirp" signal

In conclusion, computer simulation results show that the artificial neural network model is more accurate than the traditional model. The results are summarized in table 1, where the MSE (mean-square-error) and the R-squared value (also termed "coefficient of determination") are calculated and compared. In statistic, $R^{2}$ measures how well the model approximates the real data points; and an $R^{2}$ of 1.0 indicates that the model perfectly fits the data [7]:

$$
R^{2}=\frac{(\operatorname{cov}(\hat{Y}, Y))^{2}}{\sigma_{\hat{Y}} \sigma_{Y}}=\frac{(E[(\hat{Y}-\overline{\hat{Y}})(Y-\bar{Y})])^{2}}{\sigma_{\hat{Y}} \sigma_{Y}}
$$

where $\hat{Y}$ is the model output vector; $Y$ is the real system output vector; $\operatorname{cov}(\cdot)$ is the covariance function; and $\sigma(\cdot)$ represents the standard deviation. 
Table 1. The performance of the neural network and conventional model

\begin{tabular}{|c|c|c|c|c|}
\hline Signal & $\begin{array}{c}\text { Traditional } \\
\text { MSE }\end{array}$ & $\begin{array}{c}\text { NN } \\
\text { MSE }\end{array}$ & $\begin{array}{c}\text { Traditional } \\
\text { R2 }\end{array}$ & $\begin{array}{c}\text { NN } \\
\text { R2 }\end{array}$ \\
\hline $\begin{array}{c}\text { Random } \\
\text { Signal }\end{array}$ & 0.026229 & $1.9801 \mathrm{E}-6$ & 0.74866 & 0.99997 \\
\hline $\begin{array}{c}5 \text { Hz Sine } \\
\text { Wave }\end{array}$ & 0.001343 & $1.7119 \mathrm{E}-6$ & 0.92557 & 0.99988 \\
\hline $\begin{array}{c}\text { Multiple } \\
\text { Frequency }\end{array}$ & 0.0039535 & $2.2514 \mathrm{E}-6$ & 0.89522 & 0.99993 \\
\hline $\begin{array}{c}\text { Chirp } \\
\text { Signal }\end{array}$ & $6.1893 \mathrm{E}-5$ & $1.9082 \mathrm{E}-6$ & 0.85409 & 0.9921 \\
\hline
\end{tabular}

\section{CONCLUSION}

Multi gimbal systems are often used for camera stabilization in a dynamic and mobile environment. This paper investigates the applications of artificial neural networks to model the inertial characteristics (on the azimuth axis) of the inner gimbal in a gyro-stabilized multigimbal system. The neural network is trained with timedomain data obtained from gyro rate sensors of an actual camera system. The network performance is evaluated and compared with measurement data and a traditional model. Computer simulation results show the neural network model fits well with the measurement data and significantly outperforms the traditional model.

\section{REFERENCES}

[1] Hilkert, J. "Precision Line-of-Sight Control Engineering System Configuration, Analysis \& Design," in Axsys Techonologies Precision Line-of Sight Control Engineering Seminar, Grass Valley, CA, August 16-17, 2010.

[2] Hilkert, J.M, and D. Hullender, "Adaptive Control System Techniques Applied to Inertial Stabilization Systems," Proceedings from SPIE Acquisition, Tracking, and Pointing IV, vol. 1304, pp. 190-206, 1990.

[3] Olsson, H., K. J. Astrom, C. C. de Wit, M. Gafvert, and P. Lischinsky, "Friction Models and Friction Compensation," European Journal of Control, vol. 4, no. 3, pp. 176-195, 1998.

[4] D. Nguyen and B. Widrow, "Improving the learning speed of 2-layer neural networks by choosing initial values of the adaptive weights," 1990 IJCNN International Joint Conference on Neural Networks, 1990, pp. 21-26 vol.3.

[5] S. Haykin, Neural Networks: A Comprehensive Foundation, Prentice Hall, 1999.

[6] Neural Network Toolbox User's Guide, The Mathworks.

[7] http://en.wikipedia.org/wiki/Coefficient_of_determination 